\title{
When to Use Initial Triple Therapy in COPD: Adding a LAMA to ICS/LABA by Clinically Important Deterioration Assessment
}

This article was published in the following Dove Press journal: International Journal of Chronic Obstructive Pulmonary Disease

\author{
Wen-Chien Cheng (D-6 \\ Biing-Ru Wu (D) 1,3,5,6 \\ Wei-Chih Liao 1,2,4,7 \\ Chih-Yu Chen' \\ Wei-Chun Chen (1D) 1,3-6 \\ Te-Chun Hsia (iD) $1,4,8$ \\ Chih-Yen Tu ${ }^{1-3}$ \\ Chia-Hung Chen (1D) 1,2,7 \\ Wu-Huei Hsu'
}

'Division of Pulmonary and Critical Care Medicine, Department of Internal Medicine, China Medical University Hospital, Taichung, Taiwan; ${ }^{2} \mathrm{~S} c h o o l$ of Medicine, China Medical University, Taichung, Taiwan; ${ }^{3}$ Department of Life Science, National Chung Hsing University, Taichung, Taiwan;

${ }^{4}$ Department of Internal Medicine, Hyperbaric Oxygen Therapy Center, China Medical University Hospital, Taichung, Taiwan; ${ }^{5}$ Ph.D. Program in Translational Medicine, National Chung Hsing University, Taichung, Taiwan; ${ }^{6}$ Rong Hsing Research Center for Translational Medicine, National Chung Hsing University, Taichung, Taiwan; ${ }^{7}$ Graduate Institute of Clinical Medical Science, China Medical University, Taichung, Taiwan; ${ }^{8}$ Department of Respiratory Therapy, China Medical University, Taichung, Taiwan

\begin{abstract}
Purpose: Triple therapy versus dual therapy for chronic pulmonary obstructive disease (COPD) can reduce symptoms, limit the risk of acute exacerbations (AEs) as well as improve lung function. Currently, studies that feature clinically important deterioration (CID) as a composite endpoint to assess the need for treatment intensification for patients maintained on dual therapy remained to be scarce.
\end{abstract}

Patients and Methods: This study is a retrospective analysis (January 2014 to January 2018) of COPD patients that presented with moderate to severe AEs during the previous year with blood eosinophil counts $\geq 100$ cells $/ \mu \mathrm{L}$. The first line of therapy included a combination of inhaled corticosteroid (ICS) and a long-acting $\beta_{2}$ agonist (LABA). Composite CID was used in assessing the response to treatment after 24 weeks of therapy. Results: This study included 110 patients, of which 49 patients reportedly experienced CID. The most common events of CID include a decline in forced expiratory volume in 1 second (FEV1) $\geq 100 \mathrm{~mL}$ from baseline $(25 / 49,51 \%)$ and an increase in COPD Assessment Test (CAT) scores $\geq 2(13 / 49,26.5 \%)$; many of these patients respond to the addition of a longacting muscarinic antagonist (LAMA). Seven patients (7/110, 6.3\%) experienced moderate to severe exacerbations while undergoing treatment with ICS/LABA. Univariate and multivariate analyses have identified low baseline FEV1 $(\mathrm{OR}=0.81, p=0.004)$, high CAT score $(\mathrm{OR}=1.89, p=0.004)$, and the frequency of $\mathrm{AE}(\mathrm{OR}=19.86, p=0.021)$ as independent predictors of CID. A baseline FEV1 of $\leq 42 \%$, an initial CAT score $\geq 18$, and AE $\geq 2$ last year were considered the optimal cut-off values, which were identified via receiver operating characteristics (ROC) curve analysis.

Conclusion: Triple therapy (ICS/LABAs/LAMAs) may be considered as first-line treatment in patients experiencing more than 2 times moderate to severe AEs of COPD in the previous year and who have blood eosinophil counts $\geq 100$ cells $/ \mu \mathrm{L}$, reduced lung function (FEV1 $\leq 42 \%$ ), and more symptoms (CAT score $\geq 18$ ).

Keywords: inhaled corticosteroid, ICS, long-acting muscarinic antagonist, LAMA, longacting $\beta_{2}$ agonist, LABA, triple therapy, chronic pulmonary obstructive disease, COPD, clinically important deterioration, CID

\section{Introduction}

According to the 2020 Global Initiative for Chronic Obstructive Lung Disease (GOLD) report, ${ }^{1}$ pharmacological treatment for chronic pulmonary obstructive disease (COPD) should focus on a stepwise approach, with the goal of controlling symptoms and reducing exacerbations. The use of triple therapy that includes an inhaled corticosteroid (ICS), a long-acting muscarinic antagonist (LAMA), and
Correspondence: Chia-Hung Chen; ChihYu Chen

Department of Internal Medicine, China Medical University Hospital, No. 2, Yude Road, Taichung, Taiwan

Email hsnu758@gmail.com;

cychen0808@gmail.com
International Journal of Chronic Obstructive Pulmonary Disease 2020:15 3375-3384

3375

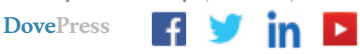


a long-acting $\beta_{2}$ agonist (LABA) had been suggested as a treatment escalation strategy. ${ }^{2}$ Several pivotal studies have reported that triple inhalation therapy can reduce the risk of exacerbations and limit symptoms, as well as improve lung function and quality of life over that observed in response to dual bronchodilator and ICS/ LABA combination therapy. ${ }^{3-7}$ Triple drug combinations can be administered using a single inhalation device; alternatively, patients may use one of two sets of devices that deliver ICS/LABA and LAMA or LABA/LAMA and ICS.

Long-acting bronchodilator combinations (LAMA/ LABA) have been identified as the initial drugs of choice for patients with more severe dyspnea, airflow obstruction, and hyperinflation. ${ }^{1,8,9}$ Likewise, the combination of ICS and LABA was considered to be a reasonable initial management strategy for subjects with a history of asthma or hospitalization for exacerbation of COPD, at least one exacerbations of COPD, and an elevated blood eosinophil counts $(\geq 100$ cells $/ \mu \mathrm{L})$ based on GOLD 2020. ${ }^{1,10}$ Meanwhile, inhaled triple therapy was reserved for patients with persistent symptoms and repeated exacerbations while undergoing dual bronchodilator (LABA/ LAMA) or ICS/LABA combination therapy. ${ }^{1,11}$ A recent study revealed that $\sim 50 \%$ of patients with COPD were escalating to triple therapy within 3 years of diagnosis. ${ }^{12}$ However, there was no evidence from randomized clinical trials that focused on the appropriate use of triple therapy as a potential first-line treatment.

Moreover, the use of a single individual measure such as forced expiratory volume in 1 second (FEV1), symptom scores, or AEs is clearly insufficient to effectively monitor COPD progression. ${ }^{13,14}$ COPD has been identified as a heterogeneous and multifactorial disease that might also be monitored using patient-reported outcomes, including decline in health status, changes in physical activity, and the frequency of COPD exacerbations. ${ }^{15}$ In order to meet this important need, the clinically important deterioration (CID) composite endpoint was developed by Mahler and Criner in 2007. ${ }^{16}$ A post hoc analysis of data from both the TORCH and ECLIPSE studies has revealed that a short-term (6 months) composite CID score is able to predict poorer long-term outcomes compared to any single CID component alone. ${ }^{17}$ The composite CID may be used to identify high-risk patients who would benefit from additional therapy, but who may have been previously regarded as low risk based on FEV1 or the absence of exacerbations alone. ${ }^{18}$ As such, early identification of these patients might be able to facilitate appropriate intensification of treatment, which in turn may limit the risk of clinical deterioration. The use of the CID composite endpoint as a prognostic measurement might also facilitate critical reductions in the size and/or length of time required to perform a meaningful clinical trial.

However, at this time, there are only very few studies providing real-world evidence with regard to the application of CID endpoints as a means to assess the potential benefit of intensified therapy for patients with COPD. Thus, the purpose of this study was to identify the clinical characteristics that predict CID in patients with moderate to severe COPD after 6 months of ICS/LABA combination treatment. Taken together, these results further suggest that inhaled triple (ICS/LABA/ LAMA) therapy might be considered as the first-line treatment in a subset of these patients in order to prevent CID.

\section{Patients and Methods}

We conducted a retrospective study at the Division of Pulmonary and Critical Care Medicine, China Medical University Hospital, Taichung, Taiwan, from January 2014 to January 2018. The study (CMUH108REC3-119) was able to secure approval from the China Medical University Hospital Institutional Review Board and informed consent was waived due to the observational and retrospective design. The data were anonymized or maintained with confidentiality.

\section{Patients and Data Collection}

Patients were considered eligible for this study if they met the following criteria: (1) age $>40$ years; (2) diagnosis of COPD based on the comprehensive clinical symptoms (cough, sputum production, and dyspnea on exertion) assessment using measures such as the COPD Assessment Test (CAT) and Modified British Medical Research Council (mMRC) questionnaire, spirometry (the presence of a post-bronchodilator FEV1/FVC < 0.7) confirms the presence of persistent airflow limitation, and a history of cigarette smoking exposure; (3) a documented history of exacerbation of the event as a medical record of worsening symptoms that required systemic or oral corticosteroids and/or antibiotics (for a moderate exacerbation) or hospitalization (for a severe exacerbation) during the 12-month period prior to screening; (4) patients with absolute eosinophil counts at or above 100 cells/ $\mu \mathrm{L}$; and (5) undergoing treatment with ICS/LABA dual combination therapy (FF/VI $100 \mu \mathrm{g} / 25 \mu \mathrm{g}$ via a Relvar Ellipta $^{\mathrm{TM}} ; \quad \mathrm{BD} / \mathrm{FF} \quad 160 \mu \mathrm{g} / 4.5 \mu \mathrm{g}$ via a Symbicort Turbuhaler $^{\mathrm{TM}}$ or Rapihaler ${ }^{\mathrm{TM}}$; FP/SAL $250 \mu \mathrm{g} / 50 \mu \mathrm{g}$ via a Seretide Accuhaler ${ }^{\mathrm{TM}}$ or Evohaler ${ }^{\mathrm{TM}}$; BDP/FF $100 \mu \mathrm{g} / 6$ 
$\mu \mathrm{g}$ via a Foster NEXThaler ${ }^{\mathrm{TM}}$ or metered dose inhaler) as a first-line therapy for COPD according to the GOLD guidelines. Patients with blood eosinophil counts less than 100 cells $/ \mu \mathrm{L}$, or those without acute exacerbation last year despite blood eosinophil counts greater than 100 cells $/ \mu \mathrm{L}$, or the duration of treatment with ICS/LABA less than 3 months, and insufficient data for analysis were excluded (Figure 1). All patients participated in healthcare case management system as a management system result into improved patient health outcomes and into healthcare quality.

\section{Treatment Assessment}

The CID composite endpoint was developed to be able to assess the likelihood of clinical deterioration; this method includes the detection of early declines in FEV1, health status assessed using the St. George's Respiratory Questionnaire (SGRQ), and the presence of exacerbations. ${ }^{19}$ However, the SGRQ is a very complicated and time-consuming measure; as such, the use of a simpler measure of health status such as COPD Assessment Test (CAT) could be advantageous. ${ }^{20,21}$ In this current study, we used CAT scores in place of the SGRQ to determine the composite CID.

The composite CID was used to assess disease status and treatment responses after 24 weeks of treatment with ICS/LABA. Parameters evaluated include the incidence of moderate or severe exacerbations, trough FEV1, and CAT scores. CID was identified using any one of the following responses: (1) decrease of $\geq 100 \mathrm{~mL}$ from baseline in trough FEV1; (2) deterioration ( $\geq 2$ units) from baseline in CAT score; and (3) a single moderate or severe exacerbation. $^{18}$

The patients in this study were then divided into two groups based on CID status (positive vs negative) at 24 weeks. Clinical data collected included age, sex, body height $(\mathrm{BH})$, body weight (BW), body mass index (BMI), smoking status, history of asthma and asthma
COPD cohort follow-up at least 6 months $(n=517)$ from 2014.01 to 2018.01
COPD cohort follow-up at least 6 months $(n=398)$ from 2014.01 to 2018.01

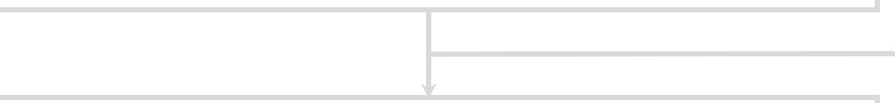

COPD cohort follow-up at least 6 months $(n=321)$ from 2014.01 to 2018.01

COPD cohort follow-up at least 6 months $(n=207)$ from 2014.01 to 2018.01

COPD cohort with $(n=110)$

1.Eosinophil counts $\geq 100$ cells $/ \mu \mathrm{L}$

2.With $A E$ in the previous year

3.ICS/LABA as first-line treatment for further analysis from 2014.01 to 2018.01
COPD Patients were excluded $(n=119)$ Eosinophil counts $<100$ cells/ $\mu \mathrm{L}$

COPD Patients were excluded $(n=77)$ Eosinophil counts $\geq 100$ cells $/ \mu \mathrm{L}$ Without $A E$ in the previous year

COPD Patients were excluded $(n=114)$

Eosinophil counts $\geq 100$ cells $/ \mu \mathrm{L}$

With $A E$ in the previous year

Treatment with ICS/LABA $<3$ months

COPD Patients were excluded $(n=97)$ Insufficient data collection

Figure I Flow diagram of patients meeting the eligibility criteria. 
control measures, pulmonary function tests (PFTs), CAT scores, modified Medical Research Council (mMRC) scores, acute exacerbation last year, the GOLD "ABCD" groups, absolute eosinophil counts, and treatment history with ICS/LABA. The GOLD "ABCD" groups are derived exclusively from patient symptoms and their history of exacerbation. (Group A: one or fewer exacerbation per year no hospitalization, mMRC $0-1$, CAT less than 10; Group B: one or fewer exacerbation per year no hospitalization, mMRC 2 or higher, CAT 10 or higher; Group C: two or more exacerbation per year one or more exacerbation with hospitalization, mMRC $0-1$, CAT less than 10; Group D: two or more exacerbation per year one or more exacerbation with hospitalization, mMRC 2 or higher, CAT 10 or higher). ${ }^{1}$

\section{Statistical Analysis}

Data were compiled and analyzed using commercial statistical software MedCalc version 15.6.1 (MedCalc, Mariakerke, Belgium). All continuous variables were reported as mean with standard deviation or median and interquartile range (IQR; 25th and 75th percentiles). Differences in continuous variables were compared using the independent $t$-tests or Kruskal-Wallis tests. Meanwhile, categorical variables were reported as the number of patients and percentages. Differences in categorical variables were examined using the F-test or chisquare test. Univariate and multivariate logistic regression analyses were used in identifying independent predictors among patients with CID. All tests of significance were two-tailed, and a $p$-value $\leq 0.05$ was considered to be statistically significant. Receiver operating characteristics (ROC) curve was used to determine the optimal cut-off values of clinical predictors of CID.

\section{Results}

In total, 110 patients were enrolled in this study, including 49 patients that experienced events associated with CID. The most common CID-associated events in our patient cohort were as follows: decline in trough FEV1 $\geq 100 \mathrm{~mL}$ from baseline $(25 / 49,51 \%)$ and deterioration in CAT scores by $\geq 2$ units $(13 / 49,26.5 \%)$. Four patients $(4 / 49$, $8.2 \%)$ were observed to have experienced two CIDassociated events, whereas seven patients $(7 / 49,14.3 \%)$ reportedly experienced a moderate or severe exacerbation during the 24 weeks on ICS/LABA combination treatment (Figure 2). The FEV1s of the patients in the $\mathrm{CID}(+)$ group $(\mathrm{n}=49)$ decreased by $105 \mathrm{~mL}(p<0.001)$. By contrast, the
FEV1s of those in the CID $(-)$ group $(\mathrm{n}=61)$ have increased significantly in response to ICS/LABA (by $85 \mathrm{~mL} ; p<0.001)$. The CAT scores of the patients in the $\mathrm{CID}(+)$ group also increased significantly ( $\geq 2$ units; $p<$ 0.001 ), while those assessed in patients in the $\operatorname{CID}(-)$ group underwent a significant decrease (1.5 units; $p<$ 0.001 ) after 24 weeks of ICS/LABA combination therapy. Among the $49 \mathrm{CID}(+)$ patients, 41 patients were treated with LAMAs (triple therapy), in addition to ongoing ICS/ LABA combination; as per our findings, the FEV1s of these patients underwent significant increases (by $130 \mathrm{~mL}$ ) in response to the addition of a LAMA ( $p<$ 0.001). The CAT scores of these patients decreased significantly (by 5 units) upon introduction of triple combination therapy $(p<0.001$; Figure 3). Table 1 includes the baseline characteristics of all patients in both the $\operatorname{CID}(+)$ and CID(-) groups. The baseline FEV1/FVC (\%), FEV1 (\%), and FEV1 (L) values of those in the $\mathrm{CID}(+)$ group were determined to be significantly lower than those reported for the $\mathrm{CID}(-)$ group, at $52.2 \%$ vs $58.3 \%$ ( $p<$ $0.001), 39.8 \%$ vs $59.5 \%(p<0.001)$, and $0.91 \mathrm{~L}$ vs 1.42 L $(p<0.001)$, respectively. The RV/TLC (\%) calculated for patients in the $\operatorname{CID}(+)$ group were also identified to be significantly higher than those in the $\operatorname{CID}(-)$ group, at $72.5 \%$ vs $65.0 \%(p<0.001)$. The patients in the $\operatorname{CID}(+)$ group had higher CAT and mMRC scores than did those in the $\operatorname{CID}(-)$ group, at 26 vs $12(p<0.001)$ and 3 vs $2(p<$ 0.001 ), respectively. The numbers of patients with $\mathrm{AE} \geq 2$ last year in the $\mathrm{CID}(+)$ group were significantly higher than those in CID (-) group, at $65.3 \%$ vs $21.3 \%$ ( $p<$ 0.001). The GOLD "ABCD" groups between these two groups were significantly different $(p=0.002)$. The proportion of patients treated with different ICS/LABA formulations was found to be similar in a comparison between the two groups $(p=0.814)$. After 24 weeks of ICS/LABA combination treatment, the FEV1s of patients in the $\mathrm{CID}(+)$ group have remained to be significantly lower than those in patients in the $\operatorname{CID}(-)$ group, at 0.82 $\mathrm{L}$ vs $1.52 \mathrm{~L}(p<0.001)$. Similarly, the CAT scores in the $\mathrm{CID}(+)$ group have also remained significantly higher than those of the $\mathrm{CID}(-)$ group, at 28 vs $11(p<0.001)$. As per current GOLD strategy, a larger fraction of the patients in Group D have reportedly experienced CID-associated events than was observed among patients in Groups A, B, or C, at $74.1 \%$ vs $22.2 \%, 40.4 \%$, or $25 \%$, respectively ( $p=0.002$; Table 1; Figure 4). No significant difference was determined between the groups with respect to age, sex, body weight $(\mathrm{BW})$, body height $(\mathrm{BH})$, body mass 


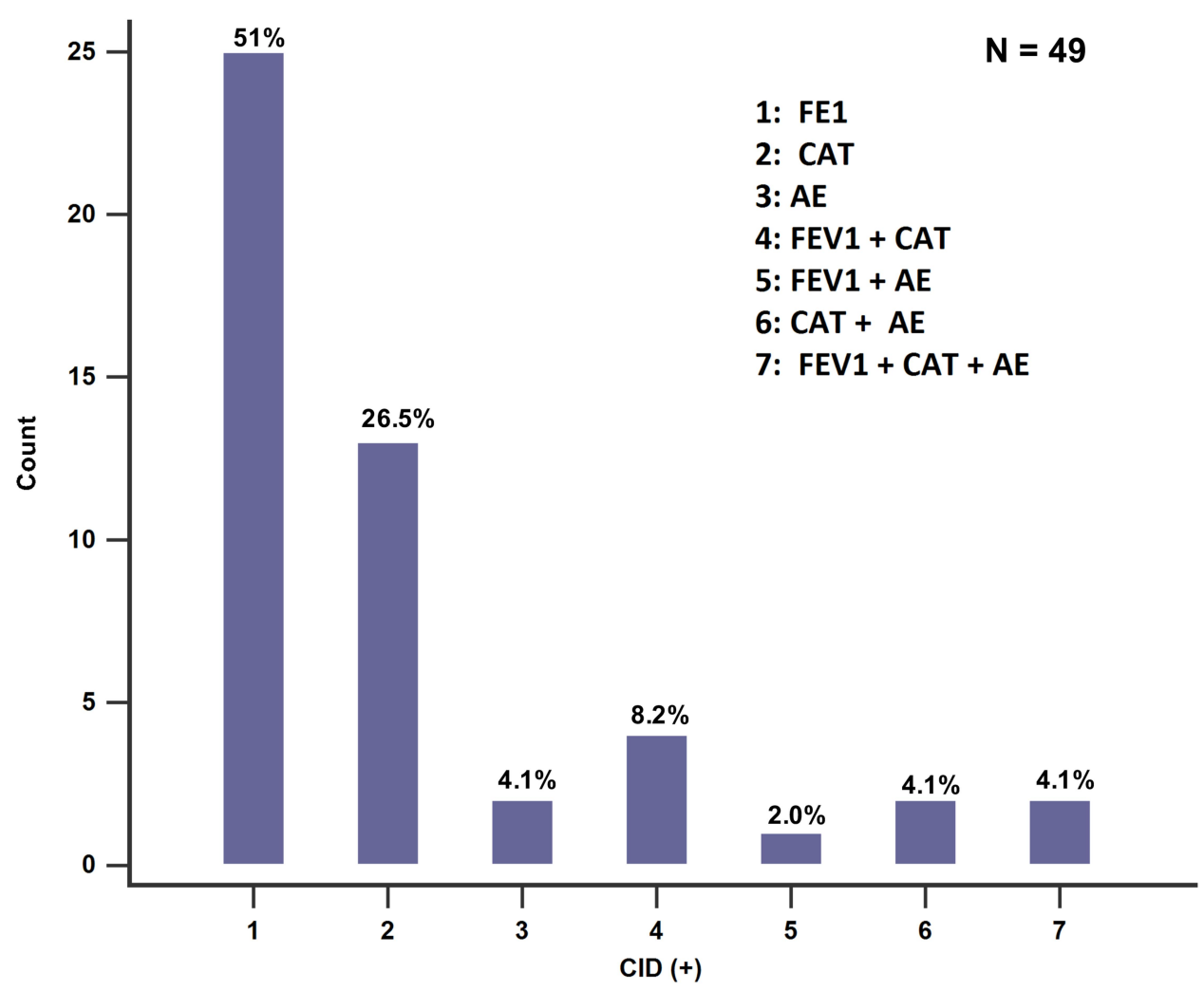

Figure 2 The proportion of CID patients in different CID events.

Abbreviations: CID, clinically Important Deterioration; FEVI, forced expiratory volume in one second (decrease of $\geq 100 \mathrm{~mL}$ from baseline in trough $\mathrm{FEVI}$ ); AE, acute exacerbation (a single moderate or severe exacerbation); CAT, chronic obstructive pulmonary disease (COPD) Assessment Test (deterioration $\geq 2$ units from baseline in CAT score).

index (BMI), smoking status or pack-years, or absolute blood eosinophil counts.

\section{Independent Predictors of CID Events}

We conducted both univariate and multivariate logistic regression analyses to determine whether any of the clinical characteristics evaluated in this study were independent predictors of CID-associated events during the 24-week study period. Univariate analysis revealed that the GOLD "ABCD" groups $(\mathrm{OR}=1.97, p<0.001)$, low FEV1 $(\mathrm{OR}=0.9, p<$ $0.001)$, high CAT score (OR $=1.29, p<0.001)$, high $\mathrm{mMRC}$ $(\mathrm{OR}=4.69, p<0.001)$, high $\mathrm{RV} / \mathrm{TLC}(\mathrm{OR}=1.08, p<0.001)$, and the frequency of exacerbation (more than two times last year) $(\mathrm{OR}=6.95, p<0.001)$ were among the potential independent predictors of CID. However, multivariate analysis identified that low FEV1 $(\mathrm{OR}=0.81, p=0.004)$ and high CAT score $(\mathrm{OR}=1.89, p=0.004)$, and the frequency of exacerbation $(\mathrm{OR}=19.86, p=0.021)$ were the only independent predictors of CID. (Table 2). We determined the optimal cut-off values to predict CID by using the ROC curve analysis (Figure 5). Taken together, our data suggest that patients with baseline FEV $1 \leq 42 \%$, initial CAT score $\geq$
18 , and $\mathrm{AE} \geq 2$ last year may be those who are likely to undergo future CID.

\section{Discussion}

To our knowledge, this is the first study that has used composite CID to identify high-risk COPD patients who might benefit from an initial triple therapy (ICS/LABA/ LAMA). Our findings suggest that patients with baseline absolute eosinophil count $\geq 100$ cells $/ \mu \mathrm{L}$, a documented history of moderate or severe AE more than 2 times last year, an initial FEV1 $\leq 42 \%$, and an initial CAT score $\geq 18$ are more likely to experience CID while under ICS/LABA combination therapy. Our results suggest that adding LAMA to ICS/LABA has resulted in significant improvements with respect to lung function and symptomatology. Inhaled triple therapy might be considered as the first-line therapy and not only on an asneeded basis to prevent CID.

However, the use of ICS for COPD treatment remains controversial. $^{22,23}$ Adverse events associated with ICS administration include pneumonia, osteoporosis, and cataracts; these findings develop in relation to the duration, 

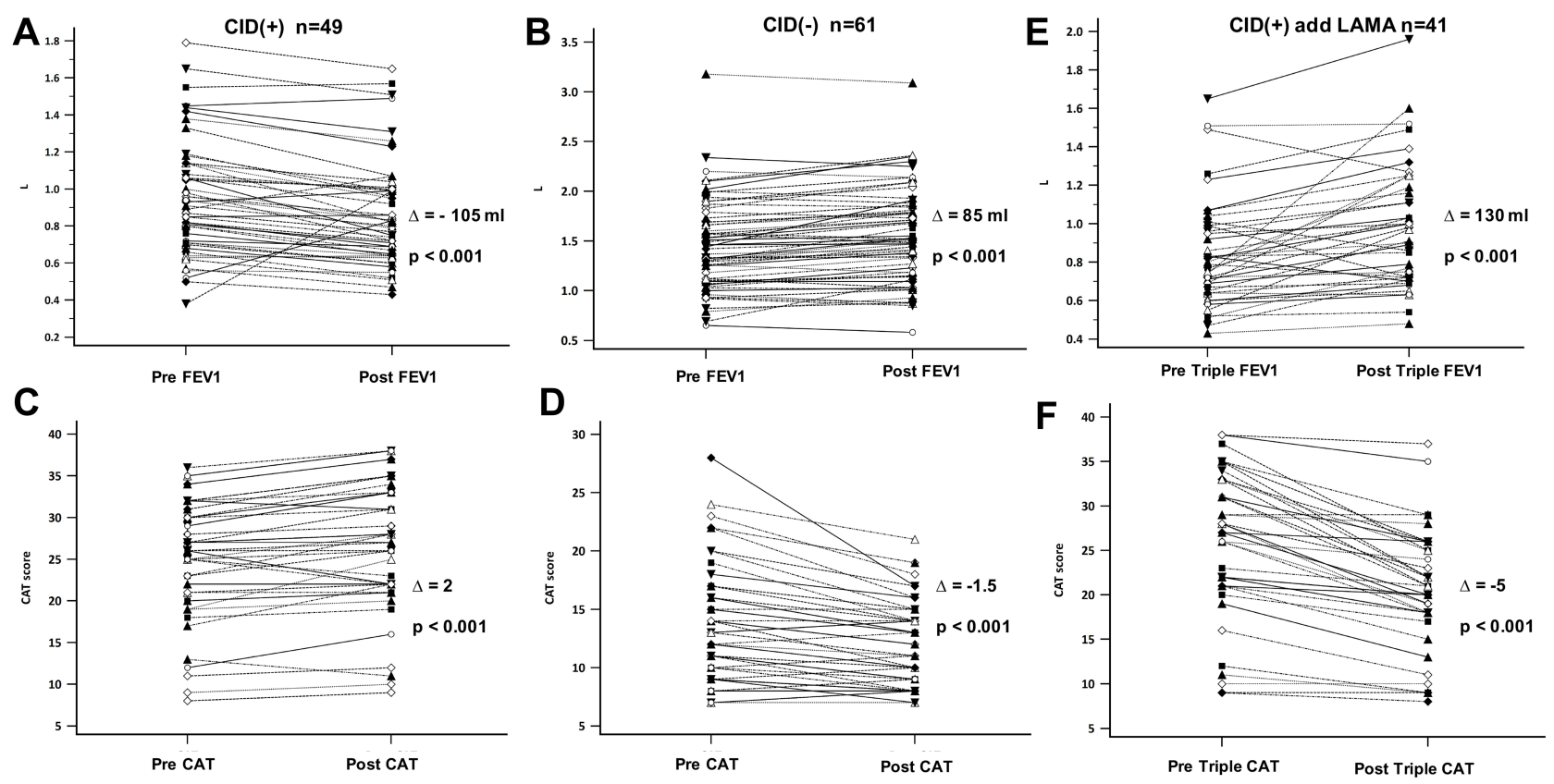

Figure 3 (A) The difference of FEVI between before and after ICS/LABA treatment in COPD patients with CID. (B) The difference of FEVI between before and after ICS/ LABA treatment in COPD patients without CID. (C) The difference of CAT score between before and after ICS/LABA treatment in COPD patients with CID. (D) The difference of CAT score between before and after ICS/LABA treatment in COPD patients without CID. (E) The difference of FEVI between before and after inhaled triple treatment in COPD patients with CID. (F) The difference of CAT between before and after inhaled triple treatment in COPD patients with CID.

Abbreviations CID, clinically important deterioration; FEVI, forced expiratory volume in one second; CAT, Chronic obstructive pulmonary disease (COPD) Assessment Test.

dose, and type of ICS treatment. ${ }^{24,25}$ The use of dual bronchodilators has changed therapeutic strategies used in treating COPD; ICS is reserved for use as an alternative combined with bronchodilators in order to prevent exacerbations. GOLD 2020 strongly recommends the use of ICS in patients with blood eosinophil counts $\geq 300$ cells $/ \mu \mathrm{L}$, history of $\mathrm{AE}$ requiring hospitalization, $\geq 2$ moderate COPD AEs per year, and a history of asthma; a threshold $\geq 100$ eosinophils $/ \mu \mathrm{L}$ can be considered in patients who have experienced at least one moderate AE. ${ }^{1}$ The patients included in our study experienced at least one moderate to severe exacerbation during the previous year, which is found to be associated with peripheral eosinophilia $(\geq 100$ cells $/ \mu \mathrm{L})$. Initial ICS/LABA combination therapy is suggested for nearly all patients with these clinical signs. After 24 weeks of treatment, only seven patients $(7 / 110,6.3 \%)$ reportedly experienced moderate or severe AEs. As such, focusing on this aspect alone, 103 patients in our study $(103 / 110,85.7 \%)$ might be considered to be under adequate control.

However, COPD is a complex, multi-factorial, and heterogeneous disease. Progression of COPD can be monitored using a variety of methods, including the rate of decline of FEV1, decline in health status, changes in physical activity, as well as the frequency of exacerbations over time. ${ }^{15}$ Characteristics of COPD have been classified into three categories, ${ }^{26,27}$ including disease severity, disease activity, and disease impact. The disease activity drives disease progression, which in turn results in an increase in disease severity and with more profound impact on quality of life. ${ }^{18,27}$ No single aspect of COPD can provide sufficient or appropriate monitoring of clinical deterioration. ${ }^{13,14,28,29}$ To address this issue, the concept of a composite CID score was developed. ${ }^{16}$ Appropriate thresholds for determining clinical deterioration include measures of minimum clinically important difference (MCID); these include a decrease of $\geq 100 \mathrm{~mL}$ from baseline trough FEV1, ${ }^{30}$ the occurrence of moderate or severe exacerbations, ${ }^{1}$ and an increase in SGRQ score $\geq 4$ units. ${ }^{31}$ The CAT was also used as an alternative to SGRQ in determining the composite CID in both the FULUIL and EMAX studies. ${ }^{20,21}$ Our study also used the CAT in place of SGRQ ins determining the composite CID.

Identification of $\mathrm{CID}(+)$ patients in the short term can also be used to predict poorer long-term prognosis. A post hoc analysis of the findings from both the TORCH and ECLIPSE studies has revealed an increased risk of mortality in patients with any evidence of CID at 6- or 12-month assessment. ${ }^{17} \mathrm{In}$ 
Table I The Baseline Clinical Factors Between COPD Patients with and without CID

\begin{tabular}{|c|c|c|c|}
\hline Clinical Factors & $\operatorname{CID}(+) n=49$ & $\operatorname{CID}(-) n=61$ & $p$-value \\
\hline Age, years & $72(63.0-79.0)$ & $72(67.0-82.3)$ & 0.154 \\
\hline Sex, male (\%) & $48(98.0)$ & $58(95.1)$ & 0.627 \\
\hline $\mathrm{BH}, \mathrm{cm}$ & $162.7(160.5-164.8)$ & $164.1(162.3-165.9)$ & 0.298 \\
\hline BW, kg & $59.2(55.7-62.6)$ & $59.8(56.6-63.1)$ & 0.770 \\
\hline BMI, $\mathrm{kg} / \mathrm{m}^{2}$ & $22.3(21.2-23.4)$ & $22.1(21.1-23.2)$ & 0.843 \\
\hline Current smoker, n (\%) & $19(38.8)$ & $17(27.9)$ & 0.227 \\
\hline Smoking, pack-years & $48.2 \pm 23.0$ & $44.2 \pm 19.6$ & 0.239 \\
\hline FEVI/FVC (\%) & $52.2(49.4-54.9)$ & $58.3(56.2-60.3)$ & $<0.001$ \\
\hline FEVI(\%) & $39.8(36.0-43.7)$ & $59.5(55.7-63.3)$ & $<0.001$ \\
\hline FEVI, L & $0.91(0.7 I-I .14)$ & $1.42(1.09-1.70)$ & $<0.001$ \\
\hline RV/TLC (\%) & $72.5(69.3-75.6)$ & $65.0(61.9-68.2)$ & 0.001 \\
\hline CAT & $26(20.8-30.3)$ & $12(9.0-16.0)$ & $<0.001$ \\
\hline $\mathrm{mMRC}$ & $3(2-4)$ & $2(I-2)$ & $<0.001$ \\
\hline Eosinophil/mL & $235(178.5-372.3)$ & $237(160.0-382.5)$ & 0.611 \\
\hline$A E \geq 2$ last year, $n(\%)$ & $32(65.3)$ & $13(21.3)$ & $<0.001$ \\
\hline Group, n (\%) & & & 0.002 \\
\hline A & $4(8.2)$ & $14(23.0)$ & \\
\hline B & $23(46.9)$ & $34(55.7)$ & \\
\hline C & $2(4.1)$ & $6(9.8)$ & \\
\hline $\mathrm{D}$ & $20(40.8)$ & $7(11.5)$ & \\
\hline ICS/LABA, n (\%) & & & 0.814 \\
\hline $\mathrm{FF} / \mathrm{VI}$ & $13(26.5)$ & $21(34.4)$ & \\
\hline FP/SAL & 14(28.6) & $14(23.0)$ & \\
\hline $\mathrm{BD} / \mathrm{FF}$ & $13(26.5)$ & $16(26.2)$ & \\
\hline BDP/FF & $9(18.4)$ & $10(16.4)$ & \\
\hline Post FEVI, L & $0.82(0.65-0.99)$ & $1.52(1.17-1.82)$ & $<0.001$ \\
\hline Post CAT & $28(22.0-33.0)$ & II (8.8-14.0) & $<0.001$ \\
\hline
\end{tabular}

Note: Continuous variables were presented as median with interquartile range. Abbreviations: $\mathrm{BH}$, body height; $\mathrm{BW}$, body weight; $\mathrm{BMI}$, body mass index; $\mathrm{CID}$, clinically important deterioration; FEVI, forced expiratory volume in one second FVC, forced vital capacity; AE, acute exacerbation; CAT, Chronic obstructive pulmonary disease (COPD) Assessment Test; mMRC, Modified Medical Research Council; ICS/LABA, inhaled corticosteroids/long-acting $\beta 2$ agonist; FF/VI, fluticasone furoate/vilanterol; FP/SAL, fluticasone propionate/salmeterol; BD/FF, budesonide/ formoterol fumarate dehydrate; BDP/FF, beclomethasone dipropionate/formoterol fumarate dehydrate; IQR, interquartile range.

view of these findings, earlier and more aggressive management of COPD may aid in reducing the risk of disease progression. ${ }^{18}$ This current study used the composite CID to assess the response to ICS/LABA combination and to identify high-risk patients who experienced CID while on this regimen. After 24 weeks on ICS/LABA combination therapy, the patients in the $\mathrm{CID}(+)$ group were observed to have lower FEV1s and FEV1/FVC\% values; they also had higher symptoms scores (CAT and mMRC) and more exacerbation last year than did those in the $\mathrm{CID}(-)$ group. As such, patients with initial FEV1 $\leq 42 \%$, CAT scores $\geq 18$, and $\mathrm{AE} \geq 2$ last year can be regarded as high-risk patients who may benefit from the addition of LAMA to their therapeutic regimens.

Of the 110 patients enrolled, 49 patients reportedly experienced at least 1 CID-associated event during the 24 -week trial of ICS/LABA combination therapy; the most common of the CID-associated events were a decline in FEV1 $\geq 100 \mathrm{~mL}$ and an increase in CAT score $\geq 2$ units. In total, 41 patients (41/49; 83\%) received additional therapy with LAMA; the FEV1s reported for these patients underwent a significant increase (by $130 \mathrm{~mL}$ ), whereas the CAT scores decreased by 5 units in response to triple inhalation therapy. The results of our study were consistent with those in previous reports. ${ }^{3-5,7}$ A metaanalysis also indicated that adding LAMA to ICS/LABA therapy did not result in an increase in serious cardiovascular events and, instead, offered significant clinical benefit to patients with COPD who had been maintained on an ICS/ LABA regimen; these benefits included improvement of trough FEV1 and prevention of AEs. ${ }^{32}$ In a post hoc analysis of the FULFIL study, triple inhalation therapy resulted in significant reductions in CID versus responses observed to ICS/LABA alone; these responses were determined to persist for a fivefold longer period without deterioration of lung function. ${ }^{20}$ Another study reported that providing a LAMA to patients maintained on ICS/LABA resulted in a $45 \%-58 \%$ reduction in CID compared to those undergoing treatment with ICS/LABA alone. ${ }^{33}$ Singh et $\mathrm{al}^{34}$ reported that triple therapy was able to reduce the risk of a first CID event compared to what was observed in response to ICS/LABA, LAMA/LABA, or LAMA monotherapy. Likewise, symptomatic COPD with asthma traits should be treated with triple therapy if not adequately controlled by ICS/LABA. ${ }^{35}$ According to previous studies, triple therapy might be recommended for COPD patients who underwent a significant decline in lung function and who developed more symptoms so as to reduce the risk of the occurrence of CID.

According to the GOLD 2020 report, ${ }^{1}$ triple therapy was recommended only for use as a step-up protocol in patients with diseases that remained uncontrolled in response to LAMA/LABA or ICS/LABA dual therapy; however, the experts who contributed to this study identified two conditions in which triple therapy might be considered as a first-line treatment. These conditions include (1) cases in which COPD was diagnosed in light of unremitting and severe exacerbations ${ }^{36}$ and (2) cases in which symptomatic patients were diagnosed with severe airflow limitation (FEV1 $<50 \%$ ), frequent moderate $(\geq 2)$ or severe AEs in the previous year, and peripheral eosinophilia $(>300 / \mu \mathrm{L}) .{ }^{36}$ Our findings are consistent with this expert advice; results from this study indicate that patients with moderate or severe AEs more than 2 times in the previous year, peripheral eosinophilia $\geq 100 / \mu \mathrm{L}$, initial severe airway obstruction (FEV1 $\leq 42 \%$ ), and multiple 


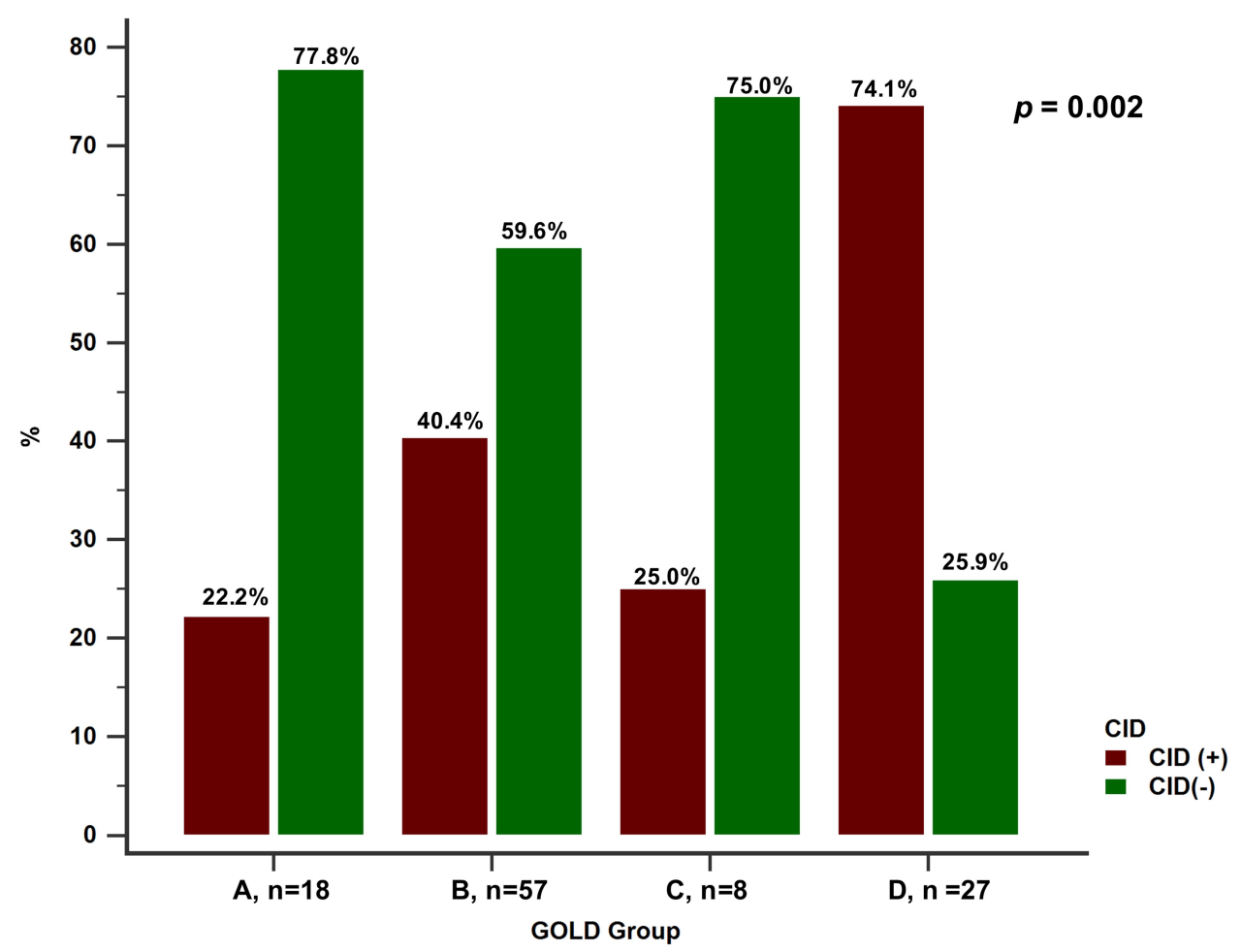

Figure 4 The proportions of patients with each GOLD classification (A--D) according to COPD patients with or without CID. Abbreviation: CID, clinically important deterioration.

significant symptoms $(\mathrm{CAT} \geq 18)$ were likely to require triple therapy to prevent the development of CID.

There were several limitations in our study. First, this study was retrospective in nature and included observational characteristics only; furthermore, the decision to treat patients with ICS/LABA was determined on an individual basis by each clinical physician. Second, our study was performed in a single institution with comparatively few patients and some degree of selection bias. Furthermore, this study

Table 2 Univariate and Multivariate Analysis of Clinical Predictors of COPD Patients with CID

\begin{tabular}{|c|c|c|c|c|}
\hline \multirow[t]{2}{*}{ Clinical Factors } & \multicolumn{2}{|l|}{ Univariate } & \multicolumn{2}{|l|}{ Multivariate } \\
\hline & OR $(95 \% \mathrm{Cl})$ & $p$-value & OR $(95 \% \mathrm{CI})$ & $p$-value \\
\hline Age, years & $0.96(0.93-1.01)$ & 0.141 & - & - \\
\hline Group & $1.97(1.32-2.95)$ & $<0.001$ & $3.75(0.86-16.37)$ & 0.077 \\
\hline Eosinophil/mL & $0.99(0.99-1.01)$ & 0.745 & - & - \\
\hline FEVI(\%) & $0.90(0.87-0.94)$ & $<0.001$ & $0.8 I(0.70-0.94)$ & 0.004 \\
\hline CAT & $1.29(1.18-1.40)$ & $<0.001$ & $1.89(1.22-2.95)$ & 0.004 \\
\hline $\mathrm{mMRC}$ & $4.69(2.65-8.31)$ & $<0.001$ & $0.11(0.01-1.06)$ & 0.056 \\
\hline $\mathrm{RV} / \mathrm{TLC}$ & $1.08(1.03-1.14)$ & 0.001 & $1.01(0.91-1.12)$ & 0.829 \\
\hline$A E \geq 2$ last year & $6.95(2.97-16.25)$ & $<0.001$ & $19.86(1.58-249.5)$ & 0.021 \\
\hline
\end{tabular}

Abbreviations: CID, clinically important deterioration; FEVI, forced expiratory volume in one second; CAT, Chronic obstructive pulmonary disease (COPD) Assessment Test; mMRC, Modified Medical Research Council; RV, residual volume; TLC, total lung capacity; $\mathrm{AE}$, acute exacerbation; $\mathrm{OR}$, odds ratio; $\mathrm{Cl}$, confidence interval. included only those individuals with at least one moderate to severe exacerbation the previous year, with blood peripheral eosinophilia ( $\geq 100$ cells $/ \mu \mathrm{L}$ ), and undergoing treatment with ICS/LABA as first-line treatment. The number of patients in our real-world study was not sufficient to perform adjusted analysis. Therefore, multivariate analysis was performed to reduce the magnitude of bias in observational retrospective study. Third, some of the patients in the CID $(+)$ group did not undergo step-up with additional bronchodilator therapy with LAMA; this has been identified as a clear limitation of this observational study. Fourth, the biomass exposure history only included cigarette smoking because it was not easy to identify the exposure to environmental agents in a retrospective study. Fifth, the proportion of male patients in our cohort was high. To balance the difference in gender in the real-world study was difficult. Sixth, we did not provide the adherence rate data. However, the poor adherence rate between these two groups (CID (+) and CID(-)) may not be significantly different because all patients participated in healthcare case management system. Despite these limitations, the current study provided the first real-world evidence suggesting that a high-risk group could be identified via an assessment of composite CID status that also focused on the potential benefits of step-up treatment in response to a CID-associated event. 


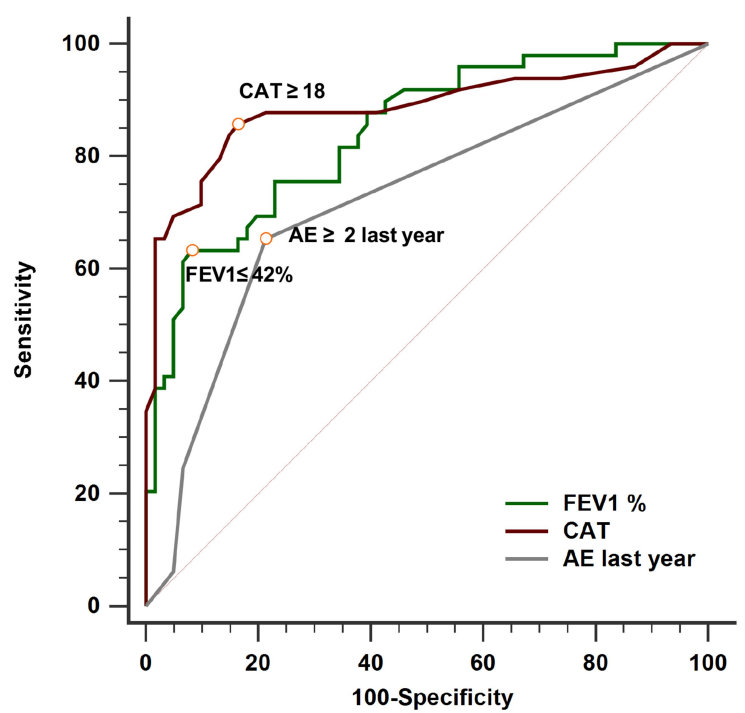

\begin{tabular}{lcr}
\hline Variable & AUC & $\mathbf{9 5 \% \mathrm { Cl }}$ \\
\hline CAT $\geq 18$ & 0.885 & 0.810 to 0.938 \\
FEV1 $\leq 42 \%$ & 0.842 & 0.761 to 0.905 \\
AE $\geq 2$ last year & 0.721 & 0.627 to 0.802 \\
\hline
\end{tabular}

Figure 5 ROC curve comparing three clinical predictors for the COPD patients with CID.

Abbreviations: CID, clinically important deterioration; FEVI, forced expiratory volume in one second; CAT, Chronic obstructive pulmonary disease (COPD) Assessment Test; AE, acute exacerbation; ROC, receiver operating characteristic curve; AUC, area under curve.

\section{Conclusion}

In conclusion, our findings revealed that an initial FEV1 $\leq 42 \%$, CAT score $\geq 18$, and the $\mathrm{AE} \geq 2$ last year were independent risk factors that predict CID in COPD patients maintained on ICS/LABA therapy, who were also identified to have experienced moderate to severe AEs during the previous year in association with peripheral blood eosinophil counts $\geq 100$ cells $/ \mu \mathrm{L}$. Adding LAMA to the ICS/LABA regimen has been determined to provide significant clinical benefit in this patient cohort. As such, triple therapy might be considered as a first-choice regimen for patients who are identified with this risk profile.

\section{Abbreviations}

LAMA, long-acting muscarinic antagonist; LABA, longacting $\beta_{2}$ agonist; ICS, inhaled corticosteroid; COPD, chronic pulmonary obstructive disease; CID, clinically important deterioration; GOLD, Global Initiative for Chronic Obstructive Lung Disease; MCID, minimum clinically important difference; mMRC, modified Medical Research Council; CAT, COPS Assessment Test; SGRQ, St George's Respiratory Questionnaire; FEV1, forced expiratory volume in one second; FVC, forced vital capacity; AE, acute exacerbation.

\section{Data Sharing Statement}

Not applicable.

\section{Ethics Approval and Consent to Participate}

The Investigational Review Board approved this review at China Medical University Hospital (CMUH108-REC3 -119). The requirement of individual patient consent was unnecessary because of the retrospective design. The data was anonymized or maintained with confidentiality.

\section{Consent for Publication}

All authors have reviewed and approved the manuscript for publication.

\section{Funding}

There is no funding to report.

\section{Disclosure}

The authors report no conflicts of interest for this work.

\section{References}

1. Global Initiative for Chronic Obstructive Lung Disease (GOLD). Global strategy for the diagnosis, management, and prevention of chronic obstructive pulmonary disease (2020 report). Avaliable from: http://goldcopd.org/.Accessed January 2020.

2. Lopez-Campos JL, Marquez-Martin E, Ortega-Ruiz F. Triple therapy vs. triple therapy in COPD. Arch Bronconeumol. 2017;53(8):419-420.

3. Ferguson GT, Rabe KF, Martinez FJ, et al. Triple therapy with budesonide/glycopyrrolate/formoterol fumarate with co-suspension delivery technology versus dual therapies in chronic obstructive pulmonary disease (KRONOS): a double-blind, parallel-group, multicentre, Phase 3 randomised controlled trial. Lancet Respir Med. 2018;6 (10):747-758.

4. Lipson DA, Barnacle H, Birk R, et al. FULFIL trial: once-daily triple therapy for patients with chronic obstructive pulmonary disease. $A m$ J Respir Crit Care Med. 2017;196(4):438-446.

5. Lipson DA, Barnhart F, Brealey N, et al. Once-daily single-inhaler triple versus dual therapy in patients with COPD. $N$ Engl J Med. 2018;378(18):1671-1680.

6. Papi A, Vestbo J, Fabbri L, et al. Extrafine inhaled triple therapy versus dual bronchodilator therapy in chronic obstructive pulmonary disease (TRIBUTE): a double-blind, parallel group, randomised controlled trial. Lancet. 2018;391(10125):1076-1084.

7. Singh D, Papi A, Corradi M, et al. Single inhaler triple therapy versus inhaled corticosteroid plus long-acting beta2-agonist therapy for chronic obstructive pulmonary disease (TRILOGY): a double-blind, parallel group, randomised controlled trial. Lancet. 2016;388 (10048):963-973.

8. Calzetta L, Rogliani P, Matera MG, Cazzola M. A Systematic review with meta-analysis of dual bronchodilation with LAMA/LABA for the treatment of stable COPD. Chest. 2016;149(5):1181-1196. 
9. Calverley PMA, Anzueto AR, Carter K, et al. Tiotropium and olodaterol in the prevention of chronic obstructive pulmonary disease exacerbations (DYNAGITO): a double-blind, randomised, parallel-group, active-controlled trial. Lancet Respir Med. 2018;6 (5):337-344.

10. Martinez FJ, Vestbo J, Anderson JA, et al. Effect of fluticasone furoate and vilanterol on exacerbations of chronic obstructive pulmonary disease in patients with moderate airflow obstruction. Am J Respir Crit Care Med. 2017;195(7):881-888.

11. Celli BR, Wedzicha JA. Update on clinical aspects of chronic obstructive pulmonary disease. $N$ Engl J Med. 2019;381 (13):1257-1266.

12. Brusselle G, Price D, Gruffydd-Jones K, et al. The inevitable drift to triple therapy in COPD: an analysis of prescribing pathways in the UK. Int J Chron Obstruct Pulmon Dis. 2015;10:2207-2217.

13. Casanova C, Aguirre-Jaime A, de Torres JP, et al. Longitudinal assessment in COPD patients: multidimensional variability and outcomes. Eur Respir J. 2014;43(3):745-753.

14. Jones PW, Agusti AG. Outcomes and markers in the assessment of chronic obstructive pulmonary disease. Eur Respir J. 2006;27 (4):822-832.

15. Halpin DM, Tashkin DP. Defining disease modification in chronic obstructive pulmonary disease. COPD. 2009;6(3):211-225.

16. Mahler DA, Criner GJ. Assessment tools for chronic obstructive pulmonary disease: do newer metrics allow for disease modification? Proc Am Thorac Soc. 2007;4(7):507-511.

17. Naya IP, Tombs L, Muellerova H, Compton C, Jones PW. Long-term outcomes following first short-term clinically important deterioration in COPD. Respir Res. 2018;19(1):222.

18. Singh D, Criner GJ, Naya I, et al. Measuring disease activity in COPD: is clinically important deterioration the answer? Respir Res. 2020;21(1):134.

19. Singh D, Maleki-Yazdi MR, Tombs L, Iqbal A, Fahy WA, Naya I. Prevention of clinically important deteriorations in COPD with umeclidinium/vilanterol. Int $J$ Chron Obstruct Pulmon Dis. 2016;11:1413-1424.

20. Naya I, Compton C, Ismaila AS, et al. Preventing clinically important deterioration with single-inhaler triple therapy in COPD. ERJ Open Res. 2018;4:4.

21. Maltais F, Bjermer L, Kerwin EM, et al. Efficacy of umeclidinium/ vilanterol versus umeclidinium and salmeterol monotherapies in symptomatic patients with COPD not receiving inhaled corticosteroids: the EMAX randomised trial. Respir Res. 2019;20(1):238.

22. Ejiofor S, Turner AM. Pharmacotherapies for COPD. Clin Med Insights Circ Respir Pulm Med. 2013;7:17-34.
23. Kaplan AG. Applying the wisdom of stepping down inhaled corticosteroids in patients with COPD: a proposed algorithm for clinical practice. Int J Chron Obstruct Pulmon Dis. 2015;10:2535-2548.

24. Kew KM, Seniukovich A. Inhaled steroids and risk of pneumonia for chronic obstructive pulmonary disease. Cochrane Database Syst Rev. 2014;(3):CD010115.

25. Morjaria JB, Rigby A, Morice AH. Inhaled corticosteroid use and the risk of pneumonia and COPD exacerbations in the UPLIFT study. Lung. 2017;195(3):281-288.

26. Agusti A, Gea J, Faner R. Biomarkers, the control panel and personalized COPD medicine. Respirology. 2016;21(1):24-33.

27. Singh D, Roche N, Halpin D, Agusti A, Wedzicha JA, Martinez FJ. Current controversies in the pharmacological treatment of chronic obstructive pulmonary disease. Am J Respir Crit Care Med. 2016;194 (5):541-549.

28. Glaab T, Vogelmeier C, Buhl R. Outcome measures in chronic obstructive pulmonary disease (COPD): strengths and limitations. Respir Res. 2010;11:79.

29. Oga T, Tsukino M, Hajiro T, et al. Multidimensional analyses of long-term clinical courses of asthma and chronic obstructive pulmonary disease. Allergol Int. 2010;59(3):257-265.

30. Calverley PM. Minimal clinically important difference-exacerbations of COPD. COPD. 2005;2(1):143-148.

31. Jones PW. St. George's Respiratory Questionnaire: MCID. COPD. 2005;2(1):75-79.

32. Calzetta L, Cazzola M, Matera MG, Rogliani P. Adding a LAMA to ICS/LABA therapy: a meta-analysis of triple combination therapy in COPD. Chest. 2019;155(4):758-770.

33. Naya IP, Tombs L, Lipson DA, Compton C. Preventing clinically important deterioration of COPD with addition of umeclidinium to Inhaled Corticosteroid/Long-Acting beta2-agonist therapy: an integrated post hoc analysis. Adv Ther. 2018;35(10):1626-1638.

34. Singh D, Fabbri LM, Vezzoli S, Petruzzelli S, Papi A. Extrafine triple therapy delays COPD clinically important deterioration vs ICS/ LABA, LAMA, or LABA/LAMA. Int $J$ Chron Obstruct Pulmon Dis. 2019;14:531-546.

35. Postma DS, Rabe KF. The Asthma-COPD Overlap Syndrome. $N$ Engl J Med. 2015;373(13):1241-1249.

36. Vanfleteren L, Ullman A, Nordenson A, Andersson A, Andelid K, Fabbri LM. Triple therapy (ICS/LABA/LAMA) in COPD: thinking out of the box. ERJ Open Res. 2019;5:1.

\section{Publish your work in this journal}

The International Journal of COPD is an international, peer-reviewed journal of therapeutics and pharmacology focusing on concise rapid reporting of clinical studies and reviews in COPD. Special focus is given to the pathophysiological processes underlying the disease, intervention programs, patient focused education, and self management protocols. This journal is indexed on PubMed Central, MedLine and CAS. The manuscript management system is completely online and includes a very quick and fair peer-review system, which is all easy to use. Visit http://www.dovepress.com/testimonials.php to read real quotes from published authors. 